\title{
ON THE CONVERGENCE PROPERTIES OF BASIC SERIES REPRESENTING CLIFFORD VALUED FUNCTIONS
}

\author{
M. A. ABUL-EZ and D. CONSTALES
}

Received 21 January 2002

\begin{abstract}
It is shown that certain classes of special monogenic functions cannot be represented by the basic series in the whole space. New definitions for the order of basis of special monogenic polynomials are given, together with theorems on the representation of classes of special monogenic functions in certain balls and at a point.
\end{abstract}

2000 Mathematics Subject Classification: 30G35, 41A10.

1. Introduction. The regular functions considered in the present paper have values in a Clifford algebra and are nullsolutions of a linear differential operator which linearizes the Laplacian (see $[4,5,6,7]$ ).

First, recall the definition of the real $2^{m}$-dimensional Clifford algebra $\mathscr{A}_{m}$ as the real algebra freely generated by the standard basis $e_{0}, e_{1}, \ldots, e_{m}$ in $\mathbb{R}^{m+1}$ subject to the conditions $e_{0}=1$ and $e_{j} e_{k}+e_{k} e_{j}=-2 \delta_{j k}$, for $1 \leq j$ and $k \leq m$ (we refer to $[4,5]$ for the basic facts on $\mathscr{A}_{m}$ ). Note that, for example, $\mathscr{A}_{0}$ is the field of real numbers, $\mathscr{A}_{1}$ is the field of complex numbers, and $\mathscr{A}_{2}=\mathbb{U}$ is the quaternionic skew field, respectively. We canonically embed $\mathbb{R}^{m+1}$ in $\mathscr{A}_{m}$. For $x \in \mathscr{A}_{m}$, Re $x$, the real part of $x$, will stand for the $e_{0}$-component of $x$ and $\operatorname{Im} x:=x-(\operatorname{Re} x) e_{0}$. We also equip $A_{m}$ with the Euclidean norm $|x|^{2}:=\operatorname{Re}(x \bar{x})$, where the conjugation : is the unique linear morphism of $\mathscr{A}_{m}$ for which $\bar{e}_{0}=e_{0}$, $\bar{e}_{j}=-e_{j}$ for $1 \leq j \leq m$, and $\overline{x y}=\bar{y} \bar{x}$ for all $x, y \in A_{m}$. As $\mathscr{A}_{m}$ is isomorphic to $\mathbb{R}^{2^{m}}$, we may provide it with the $\mathbb{R}^{2^{m}}$-norm $|a|$, and we easily see that, for any $a, b \in A_{m},|a \cdot b| \leq 2^{m / 2}|a| \cdot|b|$, where $a=\sum_{A \subseteq M} a_{A} e_{A}$ and $M$ stands for $\{1,2, \ldots, m\}$.

Suggested by the case $m=1$, call an $\mathscr{A}_{m}$-valued function $f$ in $\mathbb{R}^{m+1}$ Clifford analytic (monogenic) provided that it is annihilated by the generalized CauchyRiemann operator $D:=\sum_{j=0}^{m} e_{j}\left(\partial / \partial x_{j}\right)$, that is, $D f=0$.

The right $\mathscr{A}_{m}$-module $\mathscr{A}_{m}[x]$, defined by

$$
\mathscr{A}_{m}[x]=\operatorname{span}_{\mathscr{A}_{m}}\left\{z_{n}(x): n \in \mathbb{N}\right\}
$$

is called the space of special monogenic polynomials, where $\mathscr{A}_{m}$ is the Clifford algebra and $x$ is the Clifford variable. The polynomial $z_{n}(x)$ is defined by 
(see [2])

$$
z_{n}(x)=\sum_{i+j=n} \frac{((m-1) / 2)_{i}((m+1) / 2)_{j}}{i ! j !} \bar{x}^{i} x^{j},
$$

where, for $b \in \mathbb{R},(b)_{l}$ stands for $b(b+1) \cdots(b+l-1), \bar{x}$ is the conjugate of $x, x \in \mathbb{R}^{m+1}$, and $\mathbb{R}^{m+1}$ is identified with a subset of $\mathscr{A}_{m}$.

If $P_{n}(x)$ is homogeneous special monogenic polynomial of degree $n$ in $x$, then (see [2])

$$
P_{n}(x)=z_{n}(x) \alpha,
$$

$\alpha$ is some constant in $\mathscr{A}_{m}$, and

$$
\sup _{|x|=R}\left|z_{n}(x)\right|=C_{n}^{m+n-1} R^{n}=\frac{(m)_{n}}{n !} R^{n},
$$

where $(m)_{n} / n !=(m+n-1) ! / n !(m-1) !$.

2. Definitions. Let $\Omega$ be a connected open subset of $\mathbb{R}^{m+1}$ containing 0 , then a monogenic function $f$ in $\Omega$ is said to be special monogenic in $\Omega$ if and only if its Taylor series near zero (which is known to exist) has the form

$$
f(x)=\sum_{n=0}^{\infty} z_{n}(x) c_{n}, \quad c_{n} \in \mathscr{A}_{m}
$$

A function $f$ is said to be special monogenic on $\bar{B}(r)$ if it is special monogenic on some connected open neighborhood $\Omega_{f}$ of $\bar{B}(r)$. The set of all functions which are special monogenic on $\bar{B}(r)$ is denoted by $S M(r)$. Clearly, $S M(r)$ is a submodule of the right $\mathscr{A}_{m}$-module $M(\bar{B}(r))$ of the functions which are monogenic in a neighborhood of $\bar{B}(r)$.

The fundamental reference for special monogenic functions are [6, 7].

A set $\beta=\left\{P_{k}(x): k \in \mathbb{N}\right\}$ of special monogenic polynomials is called basic if and only if it is a base for the space $\mathscr{A}_{m}[x]$ of special monogenic polynomials, that is,

(i) every $z_{n}(x)$ can be expressed as a right $\mathscr{A}_{m}$-linear combination of elements from $\beta$. Then we have

$$
z_{n}(x)=\sum_{k=0}^{\infty} P_{k}(x) \pi_{n k}, \quad \pi_{n k} \in \mathscr{A}_{m} ;
$$

where only a finite number of terms differ from zero and $P_{k}(x)$ is given by $P_{k}(x)=\sum_{j=0}^{k} z_{j}(x) P_{k j}, P_{k j} \in A_{m}$

(ii) let $q \in \mathbb{N}$ and let $a_{0}, a_{1}, \ldots, a_{q} \in \mathscr{A}_{m}$ be such that $\sum_{0}^{q} P_{k}(x) a_{k}=0$. Then, $a_{0}=a_{1}=a_{2}=\cdots=a_{q}=0$. 
Let $N_{n}$ denote the number of nonzero $\pi_{n k}$ in (2.2). If $\limsup _{n \rightarrow \infty} N_{n}^{1 / n}=1$, the basic set $\beta$ is called a Cannon basic set, and if this condition is not satisfied, the basic set will be called general.

Let $f(x) \in S M(r)$ be given as above, then there is formally an associated basic series given by (see [2])

$$
\sum_{k=0}^{\infty} P_{k}(x) \quad\left(\sum_{n=0}^{\infty} \pi_{n k} c_{k}\right)
$$

When this associated basic series (2.3) converges normally to $f(x)$ in some domain, it is said to represent $f(x)$ in that domain. Thus, if (2.3) converges normally to $f(x)$ in $\bar{B}(R)$, then it is said that the basic series represents $f(x)$ in $\bar{B}(R)$.

Writing

$$
\omega_{n}(R)=\sum_{i} \sup _{|x|=R}\left|P_{i}(x) \pi_{n i}\right|
$$

Whittaker [8] introduced the idea of the order and type of a Cannon set in complex setting. These order and type of a Cannon basic set have been adapted to the Clifford case and introduced in [2] as follows.

The order is

$$
\omega=\lim _{R \rightarrow \infty} \limsup _{n \rightarrow \infty} \frac{\log \omega_{n}(R)}{n \log n} .
$$

If $0<\omega<\infty$, the type is

$$
\gamma=\lim _{R \rightarrow \infty} \frac{e}{\omega} \limsup _{n \rightarrow \infty} \frac{\left[\omega_{n}(R)\right]^{1 / n \omega}}{n} .
$$

As in the case of entire special monogenic functions [1, 3], a basic set will be of increase less than order $p$, type $q$, if its order $\omega$ and type $\gamma$ satisfy one of the conditions (i) $\omega<p$ and (ii) $\omega=p, \gamma<q$. The order $\rho$ and type $\sigma$ of the entire special monogenic function $f(x)=\sum_{n=0}^{\infty} z_{n}(x) c_{n}$ is given in [2] by

$$
\rho=\limsup _{n \rightarrow \infty, c_{n} \neq 0, c_{n} \neq 1} \frac{n \log n}{\log 1 /\left|c_{n}\right|}=\limsup _{r \rightarrow \infty} \frac{\log \log \sup _{|x|=r}|f(x)|}{\log r} .
$$

If $0<\rho<\infty$, then

$$
\sigma=\frac{1}{e \rho} \limsup _{n \rightarrow \infty} n\left|c_{n}\right|^{\rho / n}=\limsup _{r \rightarrow \infty} \frac{\log \sup _{|x|=r}|f(x)|}{r^{\rho}} .
$$

In $[2,3]$, the following results are proved. 
LEMMA 2.1. If the special monogenic function $f(x)=\sum_{n=0}^{\infty} z_{n}(x) c_{n}$ is such that $\sum_{n=0}^{\infty}\left|c_{n}\right| \omega_{n}(R)$ converges, then the basic series (2.3) converges normally to $f(x)$ in $\bar{B}(R)$, or the basic series (2.3) represents $f$ in $\bar{B}(R)$.

LEMMA 2.2. If $\left\{P_{n}(x)\right\}$ is a Cannon set of order $\omega$, type $\gamma$, then the associated basic series will represent all entire special monogenic functions of increase less than order $1 / \omega$ and type $1 / \gamma$ in the whole space $\mathbb{R}^{m+1}$.

LEMMA 2.3. If $\left\{P_{n}(x)\right\}$ is a Cannon set, then the necessary and sufficient condition for the basic series to represent all entire special monogenic functions of increase less than order $p$, type $q$, is that

$$
\limsup _{n \rightarrow \infty}\left(\frac{e p q}{n}\right)^{1 / p}\left\{\omega_{n}(R)\right\}^{1 / n} \leq 1
$$

for all $R \geq 0$.

We need the following lemma in the sequel.

LEMMA 2.4. If $R \geq r>0$ and $D_{n}$ is the degree of the polynomial of the highest degree in expression (2.2), then

$$
\omega_{n}(R) \leq 2^{m} N_{n}\left(D_{n}+1\right) \sqrt{\frac{(m)_{D_{n}}}{\left(D_{n}\right) !}}\left(\frac{R}{r}\right)^{D_{n}} \omega_{n}(r) .
$$

Proof. From the expression (2.4), we get

$$
\begin{aligned}
\lambda_{n}(R) & =\sum_{k=0}^{\infty} \sup _{|x|=R}\left|P_{k}(x) \pi_{n k}\right| \\
& \leq 2^{m / 2} \sum_{k=0}^{\infty} \sup _{|x|=R}\left|P_{k}(x)\right|\left|\pi_{n k}\right| \\
& \leq 2^{m / 2} \sum_{k=0}^{\infty} 2^{m / 2} \sum_{j=0}^{D_{n}} \sup _{|x|=R}\left|z_{j}(x)\right|\left|P_{k j}\right|\left|\pi_{n k}\right| .
\end{aligned}
$$

Relying on Cauchy's inequality (cf. [3]) for the special monogenic polynomial in $P_{k}(x)$, we have

$$
\left|P_{k j}\right| \leq \sqrt{\frac{k !}{(m)_{k}}}\left(\frac{\sup _{|x|=r}\left|P_{k}(x)\right|}{r^{j}}\right)=\sqrt{\frac{k !}{(m)_{k}}} \frac{M_{k}(r)}{r^{j}} .
$$


Using this inequality and the supremum of $z_{j}(x)$, we get

$$
\begin{aligned}
\omega_{n}(R) & \leq 2^{m} \sum_{k=0}^{\infty} \sum_{j=0}^{D_{n}} \frac{(m)_{j}}{j !} R^{j} \sqrt{\frac{k !}{(m)_{k}}} \frac{M_{k}(r)}{r^{j}}\left|\pi_{n k}\right| \\
& \leq 2^{m}\left(D_{n}+1\right) \frac{(m)_{D_{n}}}{\left(D_{n}\right) !}\left(\frac{R}{r}\right)^{D_{n}} \sum_{k=0}^{\infty} \sqrt{\frac{k !}{(m)_{k}}} M_{k}(r)\left|\pi_{n k}\right| \\
& \leq 2^{m}\left(D_{n}+1\right) \frac{(m)_{D_{n}}}{\left(D_{n}\right) !}\left(\frac{R}{r}\right)^{D_{n}} N_{n} \max _{k=0}^{\infty} \sqrt{\frac{k !}{(m)_{k}}} M_{k}(r)\left|\pi_{n k}\right| \\
& =2^{m}\left(D_{n}+1\right) \frac{(m)_{D_{n}}}{\left.\left(\frac{R}{r}\right)^{D_{n}}\right) !} N_{n} \sqrt{\frac{\left(D_{n}\right) !}{(m)_{D_{n}}}} M_{D_{n}}(r)\left|\pi_{n D_{n}}\right| \\
& \leq 2^{m} N_{n}\left(D_{n}+1\right) \sqrt{\frac{(m)_{D_{n}}}{\left(D_{n}\right) !}}\left(\frac{R}{r}\right)^{D_{n}} \omega_{n}(r) .
\end{aligned}
$$

Then the lemma follows.

3. Aim of the work. The purpose of this note is to study the convergence properties of a basic series representing entire special monogenic functions, not necessary in the whole of $\mathbb{R}^{m+1}$. It will be convenient now to use the following new definitions.

If $0<\rho<\infty$, then

(A) a Cannon series is said to have property $T_{\rho}$ in a closed ball $\bar{B}(R)$ if it represents all entire special monogenic functions of order less than $\rho$ in $\bar{B}(R)$.

(B) a Cannon series is said to have property $T_{\rho}$ in an open ball $B(R)$ if it represents all entire special monogenic functions of order less than $\rho$ in $B(R)$.

(C) a Cannon series is said to have property $T_{\rho}$ at the origin (i.e., when $R \rightarrow 0$ ) if it represents every entire special monogenic function of order less than $\rho$ in some ball surrounding the origin, the size of the ball being dependent on $f(x)$.

There are, as we might expect, cases in which $\omega_{n}(R)$ either

(i) tends to infinity as $R$ tends to infinity, or

(ii) is infinite for all values of $R$ greater than a certain constant, which may be zero.

In such cases, the above results (Lemmas 2.1, 2.2, 2.3, and 2.4) will no more hold and representation in the whole space of $\mathbb{R}^{m+1}$ can never occur. Thus, new definitions of the order seem to be necessary. To avoid confusion, we might as well now define Whittaker's order as the order of a Cannon set in the whole of $\mathbb{R}^{m+1}$. 
Let

$$
\Omega(r)=\limsup _{n \rightarrow \infty} \frac{\log \omega_{n}(r)}{n \log n}
$$

Since $\Omega(r)$ is an increasing function of $r$, then

$$
\begin{aligned}
& \lim _{r \rightarrow R-0} \Omega(r)=\Omega\left(R^{-}\right), \\
& \lim _{r \rightarrow R+0} \Omega(r)=\Omega\left(R^{+}\right)
\end{aligned}
$$

exist, $\Omega\left(R^{-}\right)$for $R>0$ and $\Omega\left(R^{+}\right)$for $R \geq 0$.

We define the order of a Cannon set on $\bar{B}(R)$ as equal to $\Omega(R)$ and the order in $B(R)$ as equal to $\Omega\left(R^{-}\right)$. If $R=0$ in (3.3), then $\Omega\left(0^{+}\right)$is said to be the order of a Cannon set at the origin.

Note that $\Omega\left(R^{-}\right), \Omega(R)$, and $\Omega\left(R^{+}\right)$can all be different as Example 3.1 illustrates.

EXAMPLE 3.1. Choose $a_{2}>a_{1}$ and let

$$
\begin{gathered}
P_{0}(x)=1, \\
P_{n}(x)=z_{n}(x) \quad(n \text { even }) \\
P_{n}(x)=n^{a_{1} n}+z_{n}(x)+n^{a_{2} n} \cdot z_{2 n^{2}}(x) \quad(n \text { odd }) .
\end{gathered}
$$

Then, $\Omega(r)=a_{1}$ for $r<1, \Omega(r)=a_{2}$ for $r=1$, and $\Omega(r)=\infty$ for $r>1$; that is, $\Omega\left(1^{-}\right)=a_{1}, \Omega(1)=a_{2}$, and $\Omega\left(1^{+}\right)=\infty$, and they are all different.

There is naturally a definite correspondence between Whittaker's order and the orders $\Omega\left(0^{+}\right), \Omega\left(R^{-}\right)$, and $\Omega\left(R^{+}\right)$.

The function $D_{n}$ in Lemma 2.4 is an accurate guide to such a correspondence.

4. Results of the work. Our first theorem can be stated as follows.

THEOREM 4.1. Let $\left\{P_{n}(x)\right\}$ be a Cannon set for which

$$
D_{n}=o(n \log n)
$$

Then, Whittaker's order, the order at the origin, the order in a ball, and the order on a ball are all equal.

Notice that a basic set satisfying (4.1) is also a Cannon set and Example 4.2 shows that if (4.1) is not satisfied, the theorem is no longer true. 
EXAMPLE 4.2. Choose a sequence $\mu_{1}, \mu_{2}, \ldots, \mu_{n}, \ldots$ of prime numbers and let

$$
\begin{aligned}
& P_{0}(x)=1 \\
& P_{n}(x)=1+z_{n}(x)+z_{\mu_{n}}(x), \quad n \text { is not a prime number, } \\
& P_{n}(x)=z_{n}(x), \quad n \text { is a prime number. }
\end{aligned}
$$

It is well known that if $h_{n}$ is the $n$th prime number, then $h_{n} / n \log n \rightarrow 1$ as $n \rightarrow \infty$.

Therefore, $\Omega(R)=\log R$ for $R>1$ so that, if $1<R_{1}<R_{2}$, then $\Omega\left(R_{2}\right)>\Omega\left(R_{1}\right)$. Hence, Theorem 4.1 is false if (4.1) is not satisfied.

Having now defined $T_{\rho}$ of a basic series, the order of a Cannon set at the origin, in and on a ball, we can state the theorems concerning the convergence properties of a Cannon series.

THEOREM 4.3. If $\left\{P_{n}(x)\right\}$ is a Cannon set, then the necessary and sufficient condition for the associated basic series to have

(a) property $T_{\rho}$ in $\bar{B}(R)$ is $\Omega(R) \leq 1 / \rho$,

(b) property $T_{\rho}$ in $B(R)$ is $\Omega\left(R^{-}\right) \leq 1 / \rho$,

(c) property $T_{\rho}$ at the origin is $\Omega\left(0^{+}\right) \leq 1 / \rho$.

Also, Theorem 4.1 leads to the following interesting result.

THEOREM 4.4. Let $\left\{P_{n}(x)\right\}$ be a Cannon set satisfying (4.1). If the basic series has property $T_{\rho}$ at the origin, in $B(r)$ or in $\bar{B}(r)$, then it will have property $T_{\rho}$ in the whole of $\mathbb{R}^{m+1}$, that is, the basic series will represent all entire special monogenic functions of order less than $\rho$ in the whole of $\mathbb{R}^{m+1}$.

Proof of Theorem 4.1. From Lemma 2.4, we have

$$
\omega_{n}(R) \leq 2^{m} N_{n}\left(D_{n}+1\right) \sqrt{\frac{\left(D_{n}\right) !}{(m)_{D_{n}}}}\left(\frac{R}{r}\right)^{D_{n}} \omega_{n}(r), \quad \forall R \geq r>0 .
$$

Since (4.1) is satisfied, it follows that

$$
\Omega(R) \leq \Omega(r), \quad \forall R \geq r>0 .
$$

Also, since $\omega_{n}(r)$ is an increasing function, then

$$
\omega_{n}(R) \geq \omega_{n}(r), \quad \forall R \geq r>0 .
$$

It follows that

$$
\Omega(R) \geq \Omega(r) .
$$


Combining (4.4) and (4.6), we get

$$
\Omega(R)=\Omega(r), \quad \forall R \geq r>0 .
$$

Hence, the only possibilities are

(i) $\Omega(r)$ is a finite constant $\geq 0$ for all $r>0$,

(ii) $\Omega(r)$ is infinite for all $r>0$.

So, it is evident in both cases that Whittaker's order, the order at the origin, the order in a ball, and the order on a ball are all equal. Theorem 4.1 follows.

To prove Theorems 4.3 and 4.4, we need the following two lemmas.

LEMMA 4.5. A cannon set of

(i) order $\Omega(R)$ on $\bar{B}(R)$ has property $T_{1 / \Omega(R)}$ in the closed ball $\bar{B}(R)$,

(ii) order $\Omega\left(R^{-}\right)$in the open ball $B(R)$ has property $T_{1 / \Omega\left(R^{-}\right)}$in the open ball $B(R)$,

(iii) order $\Omega\left(0^{+}\right)$at the origin has property $T_{1 / \Omega\left(0^{+}\right)}$at the origin.

LEMmA 4.6. Let $\sum$ be a Cannon series for which $\Omega\left(R^{+}\right)>1 / \lambda$ for some $\lambda>0$, $R \geq 0$. If $\rho$ is such that $1 / \lambda<1 / \rho \leq \Omega\left(R^{+}\right)$, then there is an entire special monogenic function of order $\rho<\lambda$, which $\sum$ does not represent in any ball enclosing $\bar{B}(R)$.

Proof OF LeMmA 4.5. (i) Let $\rho=\Omega(R)=\lim \sup _{n \rightarrow \infty}\left(\log \omega_{n}(R) / n \log n\right)$, and choose $\rho_{1}>\rho$, so that $\omega_{n}(R)<n^{n \rho_{1}}$ for all $n>n_{1}$. An entire special monogenic function $f=\sum_{n=0}^{\infty} z_{n}(x) c_{n}$ of order $1 / \lambda>1 / \rho,(0<\lambda<\infty)$; its Taylor coefficient will satisfy the inequality $\left|c_{n}\right|<1 / n^{n \lambda}$. Thus, $\left|c_{n}\right| \omega_{n}(R)<$ $1 / n^{n\left(\lambda-\rho_{1}\right)}$. Now, $\rho_{1}$ can be chosen very near to $\rho$, so that $\sum_{n=0}^{\infty}\left|c_{n}\right| \omega_{n}(R)$ converges. Appealing to Lemma 2.2, the basic series represents every entire special monogenic function of order $1 / \rho$ in $\bar{B}(R)$.

(ii) Suppose that $\rho=\Omega\left(R^{-}\right)=\lim _{r \rightarrow R-0} \lim \sup _{n \rightarrow \infty}\left(\log \omega_{n}(r) / n \log n\right)$. Thus, $\Omega(r) \leq \rho$ for all $0<r<R$. If $\rho_{2}>\rho$, then we have $\omega_{n}(r)<n^{n \rho_{2}}$ for all $n>n_{2}$ and all $0<r<R$.

Again, $f(x)$ of order $1 / \mu<1 / \rho(0<\mu<\infty)$ is such that its Taylor coefficient $c_{n}$ will satisfy the inequality $\left|c_{n}\right|<1 / n^{n \mu}$. Therefore, $\left|c_{n}\right| \omega_{n}(R)<1 / n^{n\left(\mu-\rho_{2}\right)}$ and $\rho_{2}$ can be chosen very near to $\rho$, so that $\sum_{n=0}^{\infty}\left|c_{n}\right| \omega_{n}(r)$ converges for all $r<R$. It follows that $\sum_{n=0}^{\infty}\left|c_{n}\right| \omega_{n}(r)$ converges for all $r<R$. It follows from Lemma 2.2 that the basic series represents every entire special monogenic function of order $<1 / \rho$ in $B(R)$.

(iii) Let $f(x)$ be an entire special monogenic function of order $v<1 / \Omega\left(0^{+}\right)$, $(0<v<\infty)$. Since $\Omega\left(0^{+}\right)<1 / v$, it follows that we can choose $r>0$ such that $\Omega(r)<1 / v$, that is, $v<1 / \Omega(r)$. Hence, from (i), the basic series represents the entire special monogenic function $f(x)$ of order $v<1 / \Omega\left(0^{+}\right)$in $\bar{B}(r)$. It follows that every entire special monogenic function of order less than $1 / \Omega\left(0^{+}\right)$can be 
represented in some ball surrounding the origin. Thus, the proof of Lemma 4.5 is completed.

PROOF OF LEMMA 4.6. A method very similar to that of Whittaker [9] can be used. The reader will not find it difficult to justify the truth of the lemma if they use the ideas of Whittaker in his paper [9].

COROLlary 4.7. Let $\sum$ be a Cannon series and let $\Omega(R)>1 / \lambda$ for some $\lambda>0, R>0$. If $\rho$ is such that $1 / \lambda<1 / \rho \leq \Omega(R)$, then there exists an entire special monogenic function of order $\rho<\lambda$ not represented by $\sum$ in $\bar{B}(R)$. The proof follows immediately from Lemma 4.6.

Proof of Theorem 4.3. (a) The condition is sufficient for, if $\rho \leq 1 / \Omega(R)$, then, by Lemma 4.5(i), the basic series will have property $T_{\rho}$ in $\bar{B}(R)$.

The condition is necessary for, if $\Omega(R)>1 / \rho$, then we can find $\rho_{1}$ such that $1 / \rho<1 / \rho_{1} \leq \Omega(R)$. Hence, by Corollary 4.7, there is an entire special monogenic function of order $\rho_{1}<\rho$ which is not represented by Cannon series in $\bar{B}(R)$.

(b) The condition is sufficient for, if $\rho \leq 1 / \Omega\left(R^{-}\right)$, so, by Lemma 4.5(ii), the basic series will have property $T_{\rho}$ in $B(R)$.

The condition is necessary for, if $\Omega\left(R^{-}\right)>1 / \rho$, then we can find $r_{1}<R$ such that $\Omega\left(r_{1}\right)>1 / \rho$. Choose $\rho_{2}$ such that $1 / \rho<1 / \rho_{2} \leq \Omega\left(r_{1}\right)$. Hence, by Corollary 4.7, there is an entire special monogenic function of order $\rho_{2}<\rho$ for which the basic series does not represent in $\bar{B}\left(r_{1}\right)$, that is, in $B(R)$.

(c) The condition is sufficient for, if $\rho \leq 1 / \Omega\left(0^{+}\right)$, then, by Lemma 4.5(iii), the basic series will have property $T_{\rho}$ at the origin.

The condition is necessary for, if $\Omega\left(0^{+}\right)>1 / \rho$, then we can find $\rho_{3}$ such that $1 / \rho<1 / \rho_{3} \leq \Omega\left(0^{+}\right)$. It follows from Lemma 4.6 that there is an entire special monogenic function of order $\rho_{3}<\rho$ not represented by the basic series in any ball enclosing the origin. Hence, Theorem 4.3 is established.

Proof OF Theorem 4.4. Suppose that the basic series has property $T_{\rho}$ in $\bar{B}(r)$. It follows that $\Omega(r) \leq 1 / \rho$. But since (4.1) is satisfied, it follows from Theorem 4.1 that $\Omega(R)=\Omega(r)$ for all $R \geq r>0$. Hence, $\Omega(R) \leq 1 / \rho$ for all $R>0$. Thus the basic series has property $T_{\rho}$ in the whole space $\mathbb{R}^{m+1}$.

Again, if the basic series has property $T_{\rho}$ in the open ball $B(r)$, then $\Omega(r-) \leq$ $1 / \rho$, that is, for some $r_{1}<r$, we have $\Omega\left(r_{1}\right) \leq 1 / \rho$. Since (4.1) is true, then, by Theorem 4.1, $\Omega(R)=\omega\left(r_{1}\right)$ for all $R \leq r_{1}$. Therefore, $\Omega(R) \leq 1 / \rho$ for all $R>0$, that is to say that the basic series has property $T_{\rho}$ in the whole space $\mathbb{R}^{m+1}$.

Finally, let the basic series possess property $T_{\rho}$ at the origin. Hence, $\Omega\left(0^{+}\right) \leq$ $1 / \rho$, so, we can choose $r>0$ such that $\Omega(r) \leq 1 / \rho$. But the basic series is subject to (4.1), so, by Theorem 4.1, $\Omega(R)=\Omega(r)$ for all $R \geq r$. It follows that $\Omega(R) \leq 1 / \rho$ for all $R>0$. Hence, the basic series has property $T_{\rho}$ in the whole space $\mathbb{R}^{m+1}$. This completes the proof of Theorem 4.4 .

REMARK 4.8. Similar results for general basic sets can be obtained. 
ACKnowledgment. D. Constales gratefully acknowledges support from Project BOF/GOA 12051598 of Ghent University.

\section{REFERENCES}

[1] M. A. Abul-Ez, Basic sets of polynomials in complex and Clifford analysis, Ph.D. thesis, State University of Gent, Belgium, 1989.

[2] M. A. Abul-Ez and D. Constales, Basic sets of polynomials in Clifford analysis, Complex Variables Theory Appl. 14 (1990), no. 1-4, 177-185.

[3] _ Linear substitution for basic sets of polynomials in Clifford analysis, Portugal. Math. 48 (1991), no. 2, 143-154.

[4] F. Brackx, R. Delanghe, and F. Sommen, Clifford Analysis, Research Notes in Mathematics, vol. 76, Pitman (Advanced Publishing Program), Massachusetts, 1982.

[5] R. Delanghe, F. Sommen, and V. Souček, Clifford Algebra and Spinor-Valued Functions. A Function Theory for the Dirac Operator, Mathematics and Its Applications, vol. 53, Kluwer Academic Publishers, Dordrecht, 1992.

[6] P. Lounesto and P. Bergh, Axially symmetric vector fields and their complex potentials, Complex Variables Theory Appl. 2 (1983), no. 2, 139-150.

[7] F. Sommen, Plane elliptic systems and monogenic functions in symmetric domains, Rend. Circ. Mat. Palermo (2) 6 (1984), 259-269.

[8] J. Whittaker, Interpolatory Function Theory, Cambridge Tracts in Mathematics and Mathematical Physics, no. 33, Cambridge University Press, London, 1935.

[9] - On effectiveness at a point, Proc. Math. Phys. Soc. Egypt 2 (1943), no. 3, 5-13.

M. A. Abul-Ez: Department of Mathematics, Faculty of Science, South Valley University, Sohag 82524, Egypt

D. Constales: Department of Mathematical Analysis, Ghent University, B-9000 GhentGalglaan 2 Ghent, Belgium 


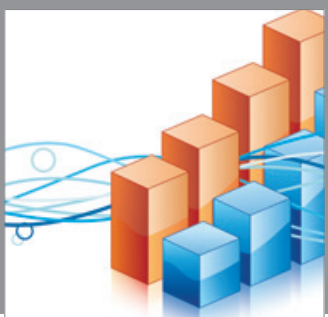

Advances in

Operations Research

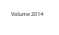

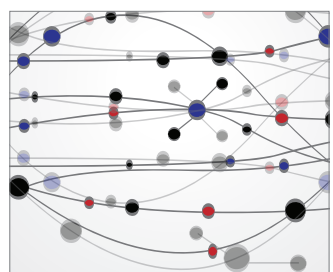

\section{The Scientific} World Journal
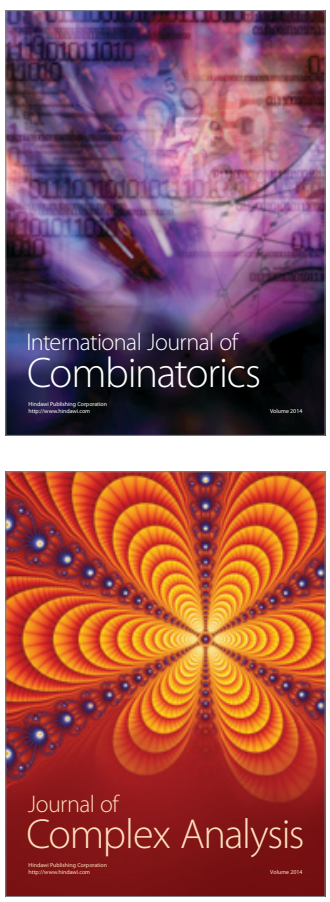

International Journal of

Mathematics and

Mathematical

Sciences
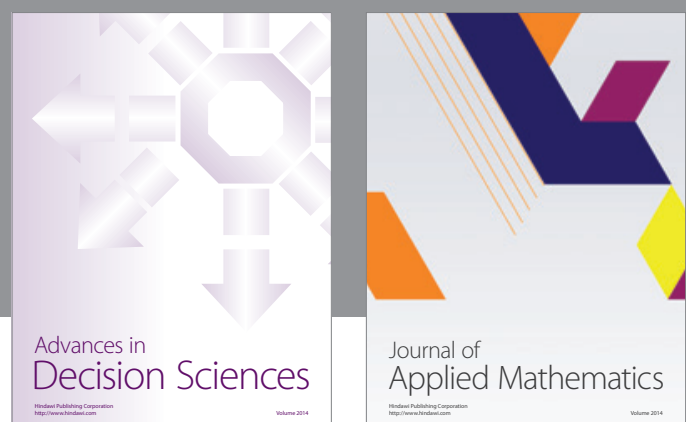

Journal of

Applied Mathematics
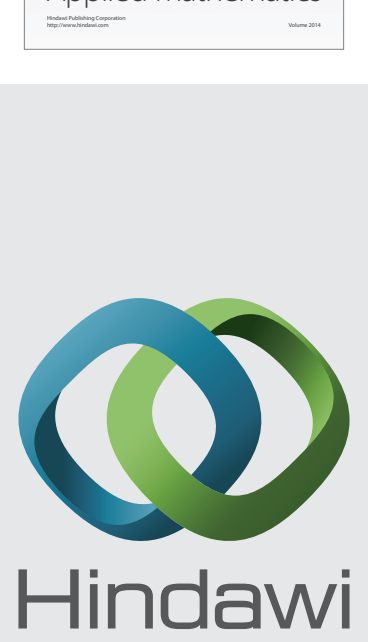

Submit your manuscripts at http://www.hindawi.com
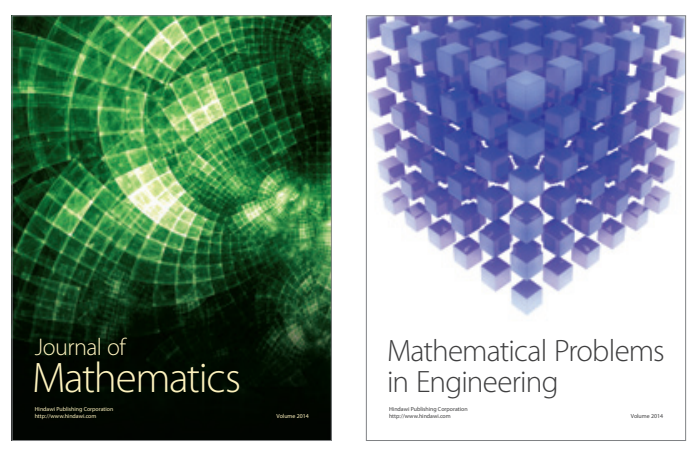

Mathematical Problems in Engineering
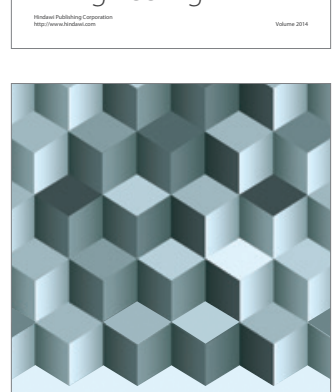

Journal of

Function Spaces
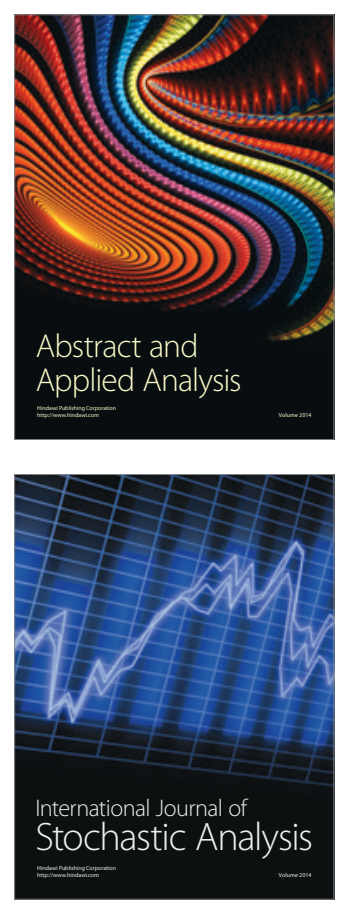

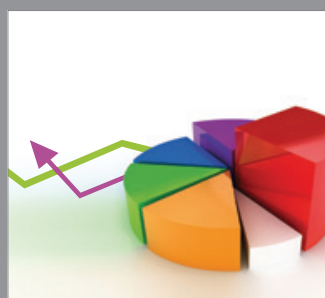

ournal of

Probability and Statistics

Promensencen
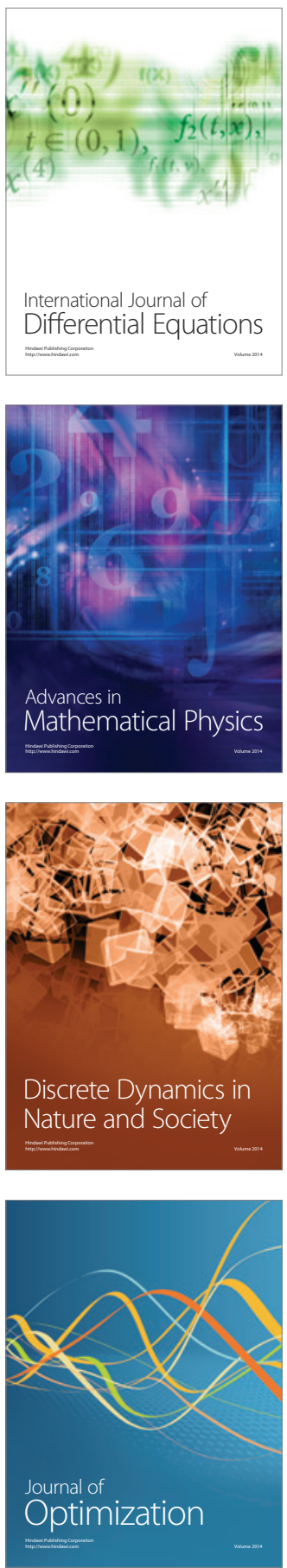